\title{
Conceptual Design of the DEMO Neutral Beam Injectors: Main Developments and R\&D Achievements
}

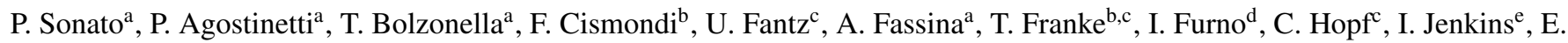

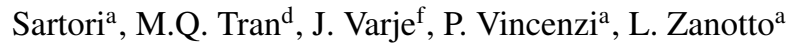 \\ ${ }^{a}$ Consorzio RFX (CNR, ENEA, INFN, Università di Padova, Acciaierie Venete SpA) Corso Stati Uniti 4 - 35127 Padova (Italy) \\ ${ }^{b}$ EUROfusion Consortium, Boltzmannstr. 2, 85748 Garching, Germany \\ ${ }^{c}$ Max-Planck-Institut für Plasmaphysik, Boltzmannstr. 2, 85748 Garching, Germany \\ ${ }^{d}$ Ecole Polytechnique Federale de Lausanne (EPFL), Swiss Plasma Center (SPC), CH-1015 Lausanne, Switzerland \\ ${ }^{e}$ Culham Centre for Fusion Energy, Science Centre, Abingdon, Oxon, OX14 3DB, UK \\ ${ }^{f}$ Department of Applied Physics, Aalto University, FI-00076 Aalto, Finland
}

\begin{abstract}
The objectives of the nuclear fusion power plant DEMO, to be built after the ITER experimental reactor, are usually understood to lie somewhere between those of ITER and a "first of a kind" commercial plant. Hence, in DEMO the issues related to efficiency and RAMI (Reliability, Availability, Maintainability and Inspectability) are among to most important drivers for the design, as the cost of the electricity produced by this power plant will strongly depend on these aspects. In the framework of the EUROfusion Work Package Heating and Current Drive (WPHCD) within the Power Plant Physics and Development (PPPT) activities, a conceptual design of the Neutral Beam Injector (NBI) for the DEMO fusion reactor has been developed by Consorzio RFX in collaboration with other European research institutes. In order to improve efficiency and RAMI aspects, several innovative solutions have been introduced in comparison to the ITER NBI, mainly regarding the beam source, neutralizer and vacuum pumping systems.
\end{abstract}

Keywords: DEMO, Neutral Beam Injector, Negative Ions, Beam Source, Conceptual Design

\section{Introduction}

DEMO (DEMOnstration Fusion Power Plant) is a proposed nuclear fusion power plant that is intended to follow the ITER experimental reactor. While in ITER the goal is to demonstrate the possibility to obtain a plasma able to sustain the fusion nuclear reaction, in DEMO the main objective is to prove the industrial feasibility of fusion by showing the electricity production from the fusion reaction, the safety aspects and the Tritium self sufficiency. The injection of high energy beams of deuterium neutrals $\left(\mathrm{D}^{0}\right)$ is one of the main tools to heat the plasma up to fusion conditions and to contribute to the plasma rotation and to the required current drive, by adopting a tangential co-injection layout of the beamlines, as shown in Fig. 1. In the framework of the EUROfusion Work Package on Heating and Current Drive (WPHCD) within the Power Plant Physics and Development (PPPT) activities, a conceptual design of the Neutral Beam Injectors (NBIs) for the DEMO fusion reactor $[1,2,3]$ has been developed by Consorzio RFX in collaboration with other European research institutes and integrated into the DEMO1 reference design.

\section{Implementation of efficiency and RAMI enhancements}

A large $\mathrm{R} \& \mathrm{D}$ effort is devoted in Europe to maximize the efficiency and obtain the most effective system with respect to the RAMI analysis meeting the requirements for the DEMO power plant. In particular with respect to the state-of-the-art negative

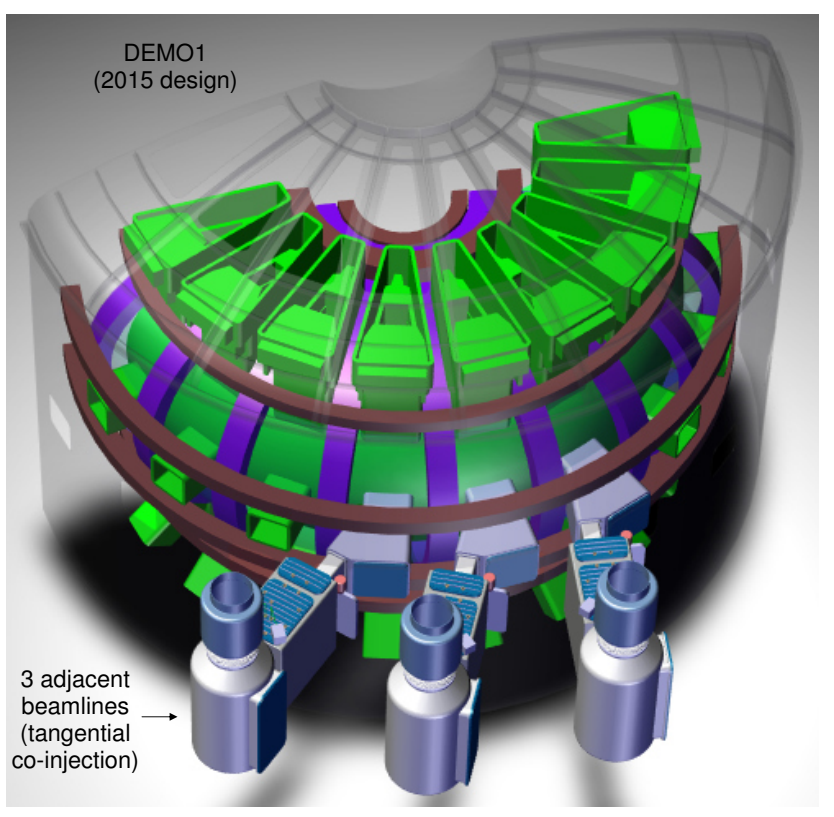

Figure 1: Integration of the Heating Neutral Beams in the DEMO1 preconceptual design. 
ion based neutral beam injector (NNBI) under construction for ITER $[4,5]$ the following aspects have been identified for efficiency and RAMI improvements.

(i) In ITER the neutralizer component is foreseen to have an efficiency of around 55\%, defined as the ratio between the power flux of neutral particles at the exit of the neutralizer and the negative ions at the entrance. To increase efficiency of the neutralizer up to $70 \%$ or more for DEMO, innovative concepts of this component, based on photo-neutralization, are being considered for DEMO. R\&D on a "closed recirculating cavity with nonlinear gating" or RING concept for the photneutralizer is currently being carried out at RFX Padova (Italy). In parallel, CEA (France) is studying another injector concept which is based on $\mathrm{D}^{-}$beam photo-neutralization in high power (MW range) resonant Fabry-Perot cavities (Siphore injector [7]), while the the HOMER experiment at IPP Garching (Germany) is dedicated to the study of the showstoppers of the photo-neutralization process [8]. The conceptual design of a neutralizer based on the RING concept has been implemented as one option in the DEMO NBI conceptual design. Nevertheless, as the Photo-Neutralizer (PN) feasibility and reliability are still to be demonstrated, the DEMO NBI has been designed to be compatible both with a PN and a gas neutralizer similar to the one foreseen for ITER. This approach aims at minimizing the development risks, by keeping more optional solutions to be selected in a later design stage.

(ii) Another possibility to increase the overall efficiency of an NBI system is to decrease the beam losses in the beam duct, which is the part that connects the NB injector with the tokamak chamber. This is particularly critical in DEMO because the duct is several meters long. To reduce re-ionization losses, the NB duct has been designed with a high performance vacuum pumping system, allowing to significantly decrease the gas pressure in that region.

(iii) The beam source for the DEMO NBI has been designed with 20 sub-sources in parallel (two adjacent columns of 10 sub-sources each) rather than a single one, following a modular design concept as shown in Fig. 2. The idea is to obtain a higher availability and resilience to fault of the individual components in each injector. The modular solution provides an optimized alignment among the corresponding apertures of the accelerator grids, since the modules have a significantly smaller size than the whole accelerator, leading to reduced horizontal and vertical deformations of the grids compared with a non-modular solution. Each sub-source features its Radio Frequency (RF) driver, designed following the research carried out by IPP Garching on the negative ion source BATMAN $[9,10]$. Alternative concepts for the ion sources are being studied at CEA (the Cybele source [7], which is based on a high power Helicon antenna developed at SPC [11]) and IPP Garching [12].

(iv) In this conceptual design, the ion beam is formed by two "blades" with large height (about $3.7 \mathrm{~m}$ ) and small width (76 $\mathrm{mm}$ ). Each of these blades is formed by 10 sub-beams, one per sub-source. The blades are strongly convergent in the vertical direction, with a fan shape. This solution is both compatible with the PN and the gas neutralizer with reduced/optimized vacuum conductance.

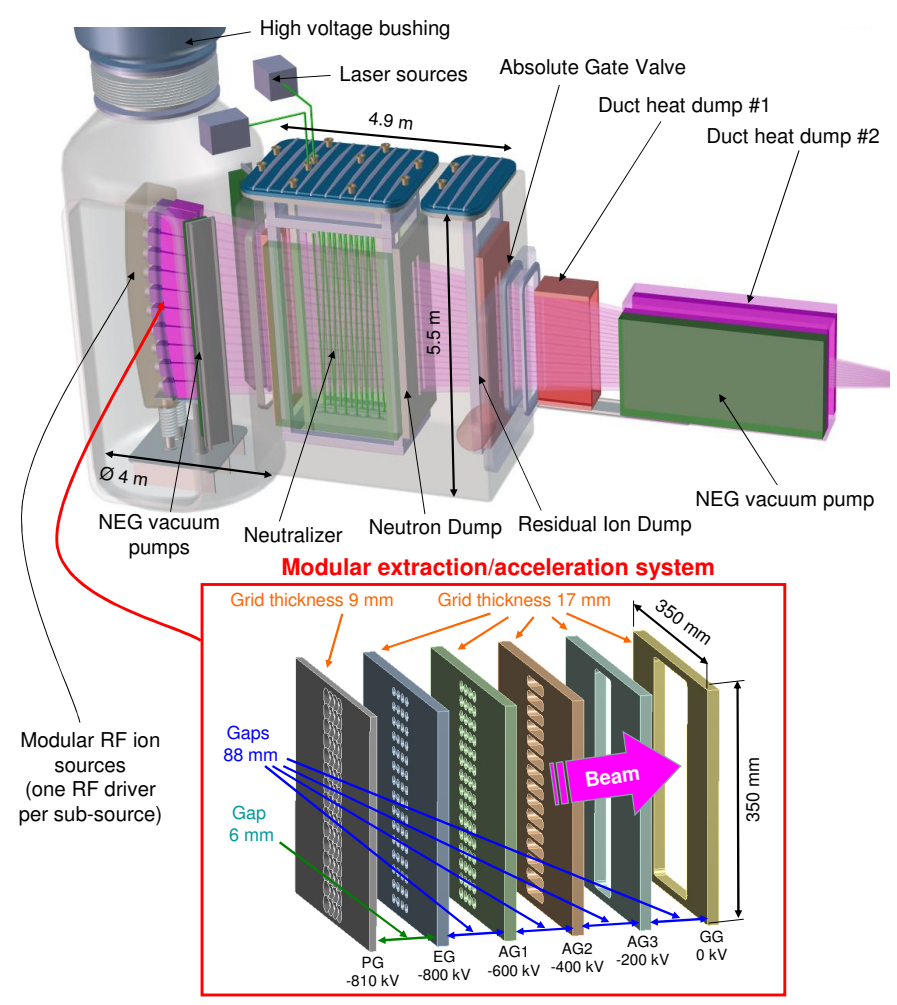

Figure 2: Overview of the DEMO NBI with the main components and a sketch of the grids of the modular extraction acceleration system.

(v) Moreover, the accelerator has been designed and optimized to maximize the vacuum pumping in order to achieve reduced stripping losses. This goal has been reached by adopting an increasing size of the apertures in the accelerator, with three shapes of the apertures (circular apertures, slotted apertures and frame-like apertures) and a design of the supporting frames that permits an optimal lateral pumping. In order to increase reliability of the vacuum pumping system, Non-Evaporable Getter (NEG) pumps are an option instead of cryopumps to provide the required vacuum pumping. This solution is under R\&D phase in RFX Padova, KIT Karlsruhe Germany and industry [13]. Compared to the cryopumps, NEG pumps present numerous advantages, i.e. they are more resistant to neutron radiation, they do not need liquid helium (and nitrogen) to maintain cryogenic temperatures and they imply a lower investment and operative cost. This solution is very attractive for the future fusion devices, but its effectiveness is under validation in present R\&D programme. An alternative solution, under development at KIT within the EUROfusion WPTFV (Work Package on Tritium Fuelling and Vacuum), is represented by the mercury diffusion pumps [14].

\section{Definition of DEMO NBI requirements}

The envisaged requirements of the DEMO NBI, agreed within the EUROfusion working group on Heating and Current Drive after several discussions, are reported in Tab. 1, where also a comparison with the ITER NBI ones is reported. 
Table 1: Main requirements and design parameters of the DEMO NBI, with a comparison to the ITER NBI. The requirements refer to the case "Advanced DEMO NBI", while the requirements for an "ITER-like DEMO NBI" are the same of the ITER NBI ones, except for the duration of the beam-on time (2 hours instead of 1 hour).

\begin{tabular}{ccc}
\hline & ITER NBI & Adv. DEMO NBI \\
\hline Requirements & $\mathrm{H}^{-} / \mathrm{D}^{-}$ & $\mathrm{D}^{-}$ \\
Species & 1000 & 800 \\
Beam energy [keV] & 40 & 34 \\
Accelerated current [A] & 0.3 & 0.2 \\
Max. ion source filling pressure [Pa] & $<7$ & $<7$ \\
Beamlet divergence [mrad] & 3600 & 7200 \\
Beam on time [s] & $<1$ & $<1$ \\
Extracted $\mathrm{e}^{-} / \mathrm{D}^{-}$fraction & not specified & $>0.70$ \\
Neutralization efficiency & & \\
Design parameters & 293 & 200 \\
Extracted current density [A m ${ }^{-2}$ ] & 0.197 & 0.185 \\
Total extraction area [m ${ }^{2}$ ] & 64.1 & 32.8 \\
Gross Power [MW] & 0.7 & 0.9 \\
Stripping/halo current losses efficiency & 40 & 33.3 \\
Accelerated current [A] & 0.95 & 0.98 \\
Beam source transmission efficiency & 0.55 & $0.7^{* *}$ \\
Neutralizer efficiency & 0.8 & 0.92 \\
Beam line/duct transmission efficiency & 16.7 & 16.8 \\
Power released to the plasma [MW] & 0.26 & 0.51 \\
Injector overall efficiency & 3 & 3 \\
Number of injectors & 50.1 & 50.4 \\
\hline Overall NBI power to the plasma [MW] & & \\
\hline
\end{tabular}

${ }^{*}$ Not a requirement for ITER; it is foreseen to be about 0.55 for ITER.

** Theoretically achievable with PN.

These requirements refer to the case "Advanced DEMO NBI", while the requirements for an "ITER-like DEMO NBI" are close to those of ITER, except for the duration of the beam on time (two hours instead of one). In fact, in this last case the DEMO NBI would be an improved version of the ITER NBI, taking into account the DEMO operating scenario and an energy recovery system for the residual ions. It can be noted that the requirements of the advanced DEMO NBI are similar but not identical to the ones of the ITER NBI. Namely, it can be observed that:

- A value of $800 \mathrm{keV}$ has been chosen for the present conceptual design of the DEMO NBI. This value is slightly decreased compared to the ITER one (1 MeV) to improve the overall reliability of the NBI system integrated into the reactor [15]. In fact, there is currently no official beam energy value specified in the DEMO requirements. On the other hand, the voltage holding of $1 \mathrm{MV}$ DC potential in presence of magnetic fields can be obtained only with optimal conditions of the electrode surfaces, in terms of smoothness and vacuum conditions.

- The maximum ion source filling pressure has been decreased from 0.3 to $0.2 \mathrm{~Pa}$, to increase efficiency. In fact, the beam losses in the accelerator are strictly linked to the gas density in the accelerator, that in turn is proportional to the pressure in the ion source.

- The maximum divergence of the beamlets must be very small (lower than 7 mrad for each beamlet) in both cases, allowing a large fraction of the particles to reach the plasma inside the main chamber.

- The required total accelerated current has been decreased of about $15 \%$, to increase availability of the NBI. In fact, high values of accelerated currents are currently obtainable only in the case of a perfect set up of the ion source, that is likely to be obtained only in particularly optimized operating conditions, especially in terms of cesium distribution inside the ion source.

- The extracted $\mathrm{e}^{-} / \mathrm{D}^{-}$fraction (or $\mathrm{e}^{-} / \mathrm{H}^{-}$fraction if the operations are with hydrogen ions) must be in both experiments kept lower than 1. A low ratio permits to limit to increase the efficiency of the extraction/acceleration system, but requires a good conditioning of the ion source, in particular regarding cesium [9]. Moreover, the thermo-structural resistance of the grids of the extraction/acceleration system is foreseen to be very critical for the DEMO NBIs, hence it is advisable to increase the safety margin against possible damages due to excessive temperature generated by the heat carried by the co-extracted electrons impinging on the grids.

- The neutralization efficiency requirement has been introduced in DEMO, differently from ITER, because it is one of the main tools to decrease the recirculating power of the plant and to increase the reliability and availability of the injector.

- The beam-on time is doubled with respect to ITER (two hours instead of one hour), to cope with a fusion power plant where the plasma pulse is two hours.

\section{Choice of the main design parameters}

Based on the requirements described in the previous section, a set of design parameters was proposed by the design team taking into account the global DEMO requirements and is reported in Tab. 1, where also the corresponding parameters of the ITER NBI are reported for comparison.

Generally, the efficiencies of the main components are increased in DEMO NBI compared to the ITER NBI values. This is achievable mainly by changing the working principle of the neutralizer (from gas to photo-neutralization), re-designing all the main components and decreasing the density of the background gas in the accelerator, in the beam line components and in the duct.

In the advanced DEMO NBI, the power consumption of the neutral beam would be approximately half than the ITER one (32.8 MW instead of 64.1 MW), while having the same nominal power injected to the plasma. This would represent a large advantage in terms of recirculating power reduction and efficiency of the whole DEMO. As a consequence, the injector overall efficiency would be about doubled in DEMO (0.51 instead of 0.26). More details on this subject are given in [16]. 


\section{Development of the conceptual design}

The present conceptual design of the advanced DEMO NBI, shown in Fig. 2, features the following main components:

- A negative ion beam source, composed of 20 sub-sources (two adjacent columns of 10 sub-sources each). The dimension of each sub-source is approximately $0.4 \mathrm{x} 0.4 \mathrm{x}$ $0.4 \mathrm{~m}^{3}$. Each sub-source features $4 \times 15$ apertures (4 in the horizontal direction, 15 in the vertical direction) with 20 horizontal x 22 vertical mm steps, like in the SPIDER [17] and MITICA [18] experiments, as well as in the beam sources of the ITER NBIs. The ion beam is formed by two "blades" with large height (about $4 \mathrm{~m}$ ) and small width (about $70 \mathrm{~mm}$ ). The blades are strongly convergent in the vertical direction, with a fan shape, to focus the entire beam to the opening in the Breeding Blanket (BB), where it enters in the main chamber.

- A PN based on the (RING) concept, but compatible with other options.

- A Residual Ion Dump (RID) featuring a flat water-cooled CuCrZr plate.

- A beam source vessel, containing the entire beam source with the related NEG pumps.

- A beam line vessel, containing the complete neutralizer and RID structures.

- A duct connecting the beam line vessel to the tokamak chamber. The duct features a large NEG pump (to reduce gas density and re-ionization losses) and two heat dumps (to dump the heat loads by re-ionization).

The proposed design solutions have been discussed with the EUROfusion groups working on the heating and current drive systems (WPHCD), on the breeding blanket (WPBB) and on the remote maintenance (WPRM), the CCFE groups studying neutronics and remote handling, and the CIEMAT group designing the BB with the Dual Coolant Lithium Lead (DCLL) concept.

\subsection{Modular solution for the beam source}

The modular solution for the beam source is found to have the following main advantages:

- A better alignment between the corresponding apertures of the grids, also in presence of thermal expansion. In fact, the thermal offsets of the apertures during the operation depend on size of the segments. In the DEMO NBI, the modular approach permits to have each module with a significantly smaller size than the whole accelerator, hence the horizontal and vertical deformations are also reduced compared with a non-modular solution. On the other hand, in the ITER NBI the modularity is only in the vertical direction (where there are four separated modules) but not on the horizontal one, introducing a quite difficult alignment and a significant thermal sensitivity for the alignment of the grid, especially in the horizontal direction [18].
- An increased neutralization efficiency, considering the present choice of RING PN, but also considering a gas neutralizer. This modular solution with two blade-like beams is the most convenient to limit the width of the neutralizer channels minimizing the gas throughput thanks to a reduced neutralizer vacuum conductance.

- A higher availability during the operations in DEMO; if some sub-sources do not work properly, the remaining ones can in any case provide the negative ion beam.

- The R\&D phase can be carried out using a small beam source, which is more flexible and less expensive than a full size prototype. Once optimized, the sub-source can be replicated to form a cluster in the DEMO NBI.

- The modular solution is also suitable for the application of other type of RF sources under development in WPHCD. In particular race track RF sources and Helicon plasma are under consideration. The application of these concepts will require an adaptation of the number of individual sub-sources still maintaining the concept of independent plasma sub-sources.

On the other hand, there are also some drawbacks:

- A more complex construction of the ion sources, because there are 20 small ion sub-sources rather than a single large one.

- A more complex construction of the extraction/acceleration system, because the 20 grid segments composing each grid must be supported by a single frame structure that has to cope with high voltage and cooling issues.

\subsection{RING type Photo-Neutralizer}

The PN, based on the RING concept [6] and shown in Fig. 3, uses two lasers with $35 \mathrm{~kW}$ power each, $1.5 \mu \mathrm{m}$ wavelength (infrared), 100 ps pulse length and $1 \mu$ s interval between pulses. By means of a second harmonic generator, only the 2nd harmonic is circulated in the PN, having a wavelength of $0.75 \mu \mathrm{m}$, half of the initial one injected by the laser. The 2 nd harmonic remains trapped in the mirror system, given by a certain number of upper/lower mirrors ( 6 in the presented design) and additional 4 mirrors with a $45^{\circ}$ angle. The negative ion beam must have enough intersection (limited to $76 \mathrm{~mm}$ for laser technological limitations). Additionally the laser has to intercept the beam in a sufficient number of times (14 in the presented design), so that a suitable neutralization power of the ion beam can be provided.

In order to have a sufficient precision on the position of the mirrors, a double frame design is proposed. The internal structure supports the laser optical systems, while the external structure supports the other auxiliary components, subjected to significant thermal loads (electron dump, neutron dump and NEG pumps). The electron dump stops the electrons accelerated together with the negative ions out of the accelerator, while the neutron dump stops the neutrons coming from the tokamak 

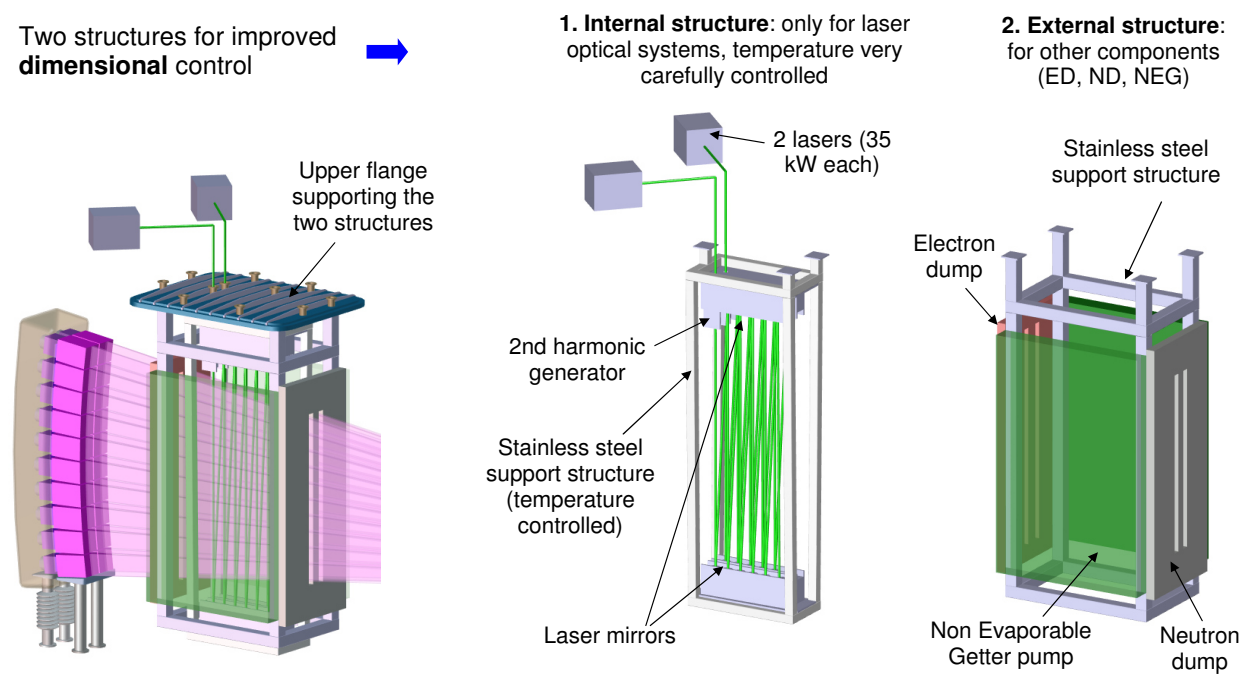

Figure 3: Neutralizer conceptual design.

chamber. The NEG pumps keep a high vacuum in the region minimizing the gas density inside the beam line vessel and consequently the stripping and re-ionization losses. In fact, the density of background gas can be much lower than with gas neutralizer, because the efficiency of the neutralizer with photoneutralization is not depending on the background gas density. This represents a significant advantage of the PN with respect to the gas neutralizer. The connection of the optical systems to the upper flange permits to regulate the mirrors when they are outside the beam line vessel and then put the system in the operating position. The dedicated cooling for the internal structure permits to carefully control the temperature of this structure during operation. On the other hand, the thermal expansion of the external structure is not so delicate hence; all the high heat load components are mounted on this structure.

\subsection{Interface with the breeding blanket}

The interface between the NBI and the BB is critical for several reasons:

- The mechanical integrity of the Breeding Blanket large segments and the reduction of TBR (Tritium Breeding Ratio) due to the presence of the NBI opening on the BB. The impact on these two aspects can be minimized by focusing the neutral beam at the center of the opening into the $\mathrm{BB}$, thus minimising the volume to be taken out from the BB structure and breeding zone. The aperture size in the blanket (height $\mathrm{x}$ width) has been reduced from the ITER values of $1060 \times 550 \mathrm{~mm}^{2}$ to $700 \times 700 \mathrm{~mm}^{2}$ of DEMO. Considering the larger height of the DEMO beam (due to the double blade shape), a significant effort has been devoted to minimize as much as possible the aperture size in DEMO. In fact, if the beam was not focused on the BB but on the tangential point in the plasma (like it is in ITER), the aperture size should have been at least $2000 \times 700 \mathrm{~mm}^{2}$.
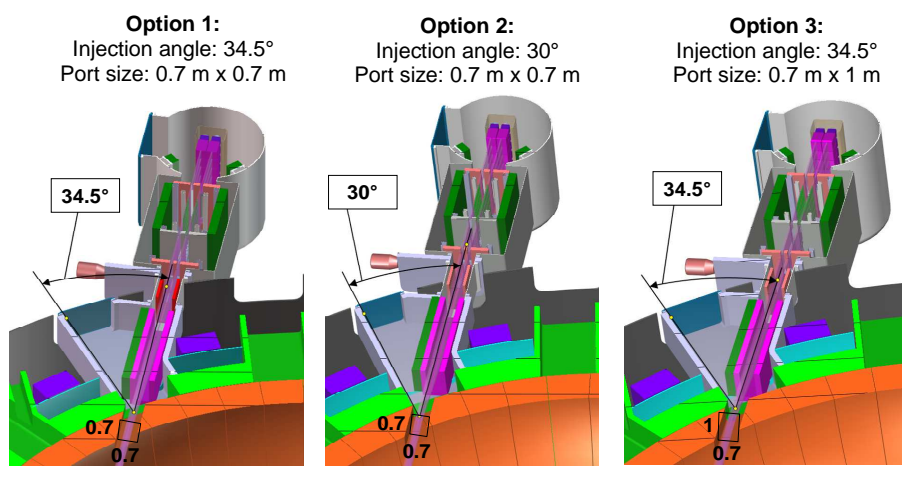

Figure 4: Interface with breeding blanket: comparison between the three design options. Option 2 is the selected one.

- The amount of neutron radiation reaching the NBI components from the tokamak, depending on the dimension of the NBI aperture and on the injection angle.

- The interaction of the beam with the plasma (and the breeding blanket first wall, in case of shine-through) in the vacuum chamber, depending on the injection angle.

- The layout of the port region, that is critical because of the interface between several critical components, like BB, NBI, Toroidal Field (TF) coils, Vacuum Vessel (VV) etc.

For these reasons, also during the conceptual phase of the design a first study of integration of NBIs and BB have been carried out. To make this the DCLL BB have been considered; this choice is arbitrary and made only to perform these evaluations.

In particular, three design options have been proposed, as shown in Fig. 4). After preliminary evaluations performed with the other involved working groups, option 2 was identified as the reference design. In fact, thanks to the reduced injection angle of $30^{\circ}$, this option requires no modification of the $\mathrm{VV}$ 

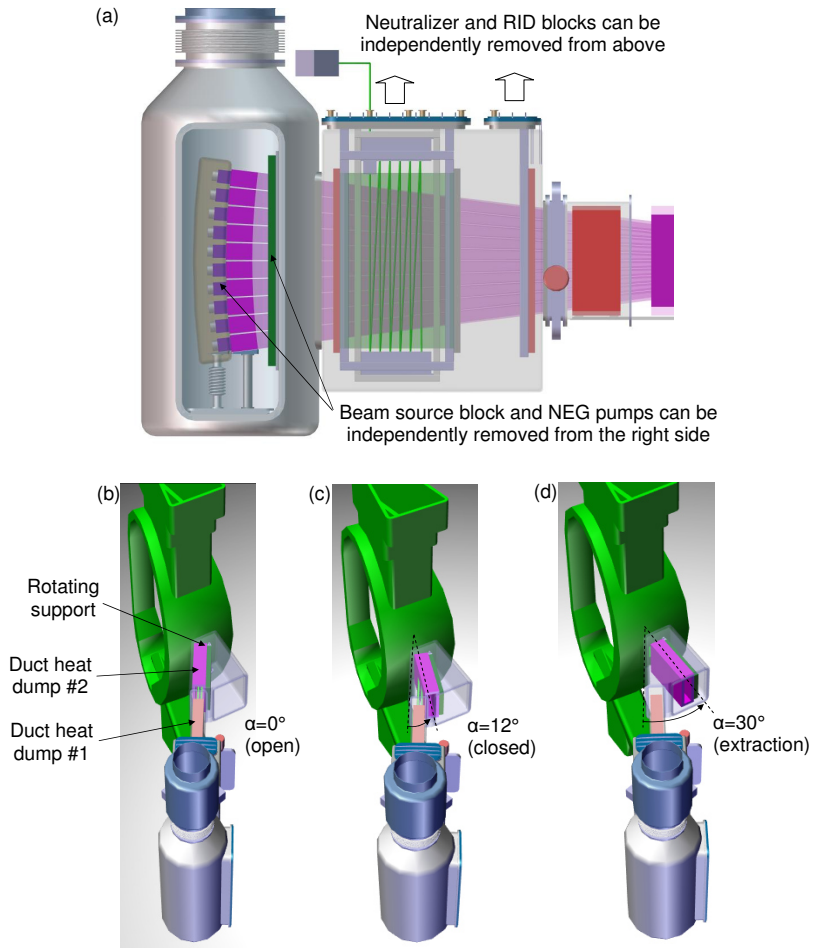

Figure 5: Maintenance strategy: (a) maintenance of the beam source and beam line components; (b) duct heat dump \#2 in the open position; (c) duct heat dump \#2 in the closed position; (d) duct heat dump \#2 in the extraction position.

due to the reduced injection angle. For the same reason, the clearance with the TF coils is larger. On the other hand, the neutronic load on the NBI components are about $12 \%$ larger for option 2 compared to option 1. This aspect is considered negligible considering the significant advantages of option 2 .

The influence of the injection angle on the interaction between the injected neutral beam and the plasma inside the tokamak and the variation of the TBR are under evaluation. The integration study into the DCLL confirms that the poloidal continuity of the BB segment can be maintained, thus keeping the same BB fixations to the VV and the same thermo-hydraulic design of the manifolds as for the other segments. Detailed studies including the thermo-hydraulic design of the lateral walls of the NBI duct into the BB has been started after establishing an initial set of boundary conditions for the heat loads imposed by the NBI in the duct. A neutronic assessment is on-going for evaluating the impact on the TBR and the neutronic heat loads on NBI and surrounding systems. A neutronic assessment is ongoing for evaluating the impact on the TBR and the neutronic heat loads on NBI and surrounding systems, in particular the TF coils.

\subsection{Maintenance strategy}

To increase the maintainability of the fusion power plant, the DEMO NBI has been designed in such a way that all the main components can be substituted without removing other components. For example, the beam source can be removed from the lateral opening of the beam source vessel (see Fig. 5a), the neutralizer and the residual ion dump from the dedicated upper flanges (see Fig. 5a), and the duct components from the radial port close to the NBI tangential port (see Fig. $5 \mathrm{~d}$ ).

The duct heat dump \#2 is in a ideal position to measure the power of the beam. In fact, it is very close to the entrance point of the beam into the main chamber, hence the measurement can be very precise. Moreover, this component is already equipped with cooling water to dump the heat from the re-ionized particles. Hence, a rotating tool is here proposed, able to rotate the duct heat dump \#2 in three positions:

- Open position, with $\alpha=0^{\circ}$ and shown in Fig. 5b, where the beam can pass through the duct heat dump \#2, while only the particles generated by re-ionization reactions are dumped on the internal walls of the duct heat dump \#2.

- Closed position, with $\alpha=12^{\circ}$ and shown in Fig. 5c, where the beam is completely intercepted by the left wall of the duct heat dump, that will be equipped with high heat flux (HHF) elements, like hypervapotrons or swirl tubes. As the angle between the beam and the left wall of the duct heat dump \#2 is small, the beam will impinge on a large area and consequently the deposited power density can be kept to relatively low values. In this position, the left wall of the duct heat dump \#2 acts like a calorimeter.

- Extraction position, with $\alpha=30^{\circ}$ and shown in Fig. 5d, where the duct heat dump \#2 is aligned with the radial direction and can be extracted for maintenance using the radial port.

\subsection{Impact on DEMO plasma}

The ionization of the neutral beam from a single injector in flat-top condition has been simulated by the Monte Carlo code BBNBI [19], using $10^{5}$ test particles. BBNBI creates the test particles starting from a detailed description of each beamlet of the injector and calculates the ionization pattern of the neutral beam in a background plasma, as shown in Fig. 6a. DEMO plasma parameters for these simulations are those of DEMO1 2015 , described in [2]. A top view of the torus with the ionization traces for NBI options 1 and 2 is shown in Fig. $6 \mathrm{~b}$ and Fig. 6c, respectively. The two different injection angles are clearly visible, with the beam aiming more on-axis for option 1. From these simulations it results that all the test particles are ionized inside the plasma, i.e. there is no shine-through neither on internal nor on external wall for both options. Only few particles are ionized in the final part of the beam trajectory inside the plasma. Considering the numerical weight of each test particle (each particle corresponds to $1.31 \times 10^{15}$ particles/s), we can conclude that the shine-through is at least less than $168 \mathrm{~W}$ (power associated to 1 test particle), i.e. $<0.001 \%$ of the NB power. The absence of shine-through can be explained by the lowered NB energy (800 keV) with respect to ITER NBI (1 $\mathrm{MeV})$, but at the same time with a higher plasma volume for DEMO. This ensures the machine wall safety for standard flattop conditions.

The different injection angles may have some effect on power deposition or on driven current density, although probably not significant for the scenario balance: the total NBI power for 


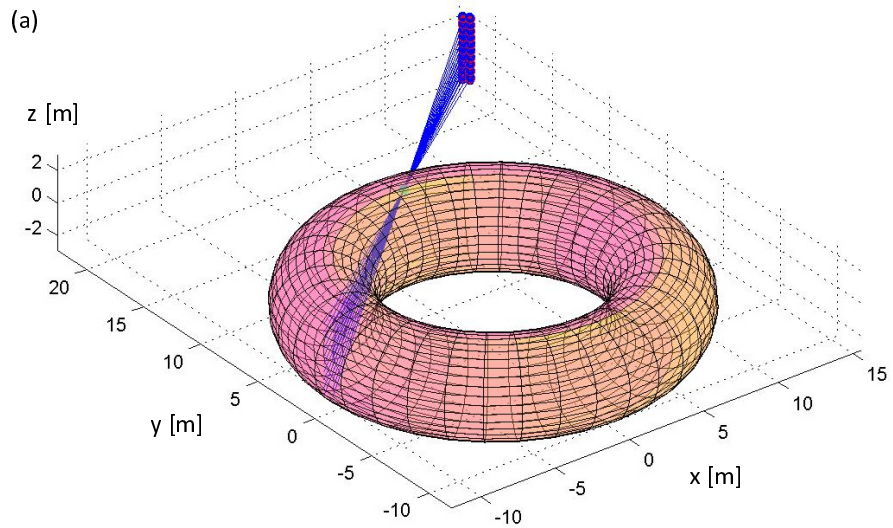

(b)

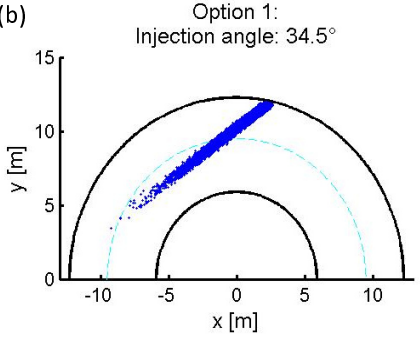

(c)

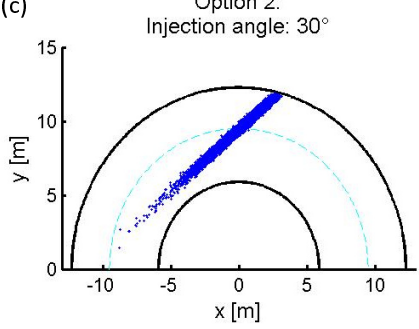

Figure 6: Investigations on the ionization of the neutral beam in the main chamber: (a) 3D view of the model with the beam trajectories (option 2); (b) ionization pattern with option 1 ; (c) ionization pattern with option 2. Black line is the wall, dotted cyan line is the plasma magnetic axis.

DEMO is indeed $50 \mathrm{MW}$ with respect to about $400 \mathrm{MW}$ of alpha heating power and the NB current drive represents less than $10 \%$ of the total plasma current in stationary conditions [2]. Further studies on DEMO scenario and NBI-plasma interaction physics are ongoing.

\section{Conclusions}

The conceptual design of the DEMO NBI has been developed in the framework of the EUROfusion activities on the heating and current drive (HCD) systems aiming to increase the overall efficiency $(>50 \%)$ and better coping with RAMI requirements. The design features a modular approach for the beam source and is ready to adopt the photo-neutralisation concept.

For a smooth integration, all the proposed design solutions have been discussed with the EUROfusion groups working on the heating and current drive systems, on the breeding blanket and on the remote maintenance, the CCFE groups studying neutronics and remote handling, and the CIEMAT group designing the Breeding Blanket. The main components of the DEMO NBI have been drafted, based on the present knowledge and on the R\&D currently being carried out in various European Research Institutes. The design of most components will be further studied and developed within EUROfusion.

In future, the various NB injector concepts under development (ITER-like and DEMO-like), could be tested, after demonstrating their feasibility and performances in the differ- ent facilities throughout Europe, in the neutral beam test facility PRIMA hosted in Padova (Italy).

\section{Acknowledgements}

The authors would like to thank A. Simonin of CEA, S. Zheng of CCFE and A. Valentine of CCFE for the fruitful discussions during the development of the conceptual design.

This work has been carried out within the framework of the EUROfusion Consortium and has received funding from the Euratom research and training programme 2014-2018 under grant agreement No 633053. The views and opinions expressed herein do not necessarily reflect those of the European Commission.

\section{References}

[1] GIRUZZI G., et al., Modelling of pulsed and steady-state DEMO scenarios, Nucl. Fusion 55 (2015) 073002.

[2] WENNINGER R., et al., The physics and technology basis entering European system code studies for DEMO, Nucl. Fusion 57 (2017) 016011.

[3] FRANKE T., et al., On the Present Status of the EU DEMO H\&CD Systems, Technology, Functions and Mix, poster SP13-63 presented at 26th SOFE conference, Austin, Texas USA (2015).

[4] TOIGO V., et al., Progress in the realization of the PRIMA Neutral Beam Test Facility, Nucl. Fusion 55 (2015) 083025.

[5] MARCUZZI D., et al., Final Design of the Beam Source for the MITICA Injector, Rev. Sci. Instrum. 87, 02 B309 (2016).

[6] FASSINA A., et al., Feasibility Study of a NBI Photoneutralizer Based on Nonlinear Gating Laser Recirculation, Rev. Sci. Instrum. 87, 02B318 (2016).

[7] SIMONIN A., et al., R\&D around a photoneutralizer-based NBI system (Siphore) in view of a DEMO Tokamak steady state fusion reactor, Nucl. Fusion 55 (2015) 123020.

[8] RAUNER D., et al., Comparison of measured and modelled negative hydrogen ion densities at the ECR-discharge HOMER, AIP Conf. Proc. 1655, 020017 (2015).

[9] FANTZ U., et al., Physical performance analysis and progress of the development of the negative ion RF source for the ITER NBI system, Nucl. Fusion 49 (2009) 125007.

[10] KRAUS W., et al., Long pulse large area beam extraction with a rf driven $\mathrm{H}^{-} / \mathrm{D}^{-}$source, Rev. Sci. Instrum. 79, $02 \mathrm{C} 108$ (2008).

[11] FURNO I., et al., A novel helicon plasma source for negative ion beams for fusion, in preparation for Nucl. Fusion.

[12] BRIEFI S. and FANTZ U., Investigation of Helicon discharges as RF coupling concept of negative hydrogen ion sources, AIP Conf Proc. 1515, 278-283 (2013).

[13] BONUCCI A., MANINI P., SIVIERO F., SONATO P., Getter pumping system, WO Patent App. PCT/IB2015/054,728 (2015).

[14] GIEGERICH T. DAY C., Conceptuation of a continuously working vacuum pump train for fusion power plants, Fusion Eng. Des. 88 (2013) 2206-2209.

[15] PILAN N., et al., Magnetic Field Effect on Voltage Holding in the MITICA Electrostatic Accelerator, IEEE Tran. Pla. Sci. Vol. 42 No.4 (2014).

[16] SONATO P., et al., Conceptual design of the beam source for the DEMO NBIs, accepted for publication in New Journal of Physics (2016).

[17] AGOSTINETTI P., et al., Physics and engineering design of the Accelerator and Electron Dump for SPIDER, Nucl. Fusion 51 (2011) 063004.

[18] AGOSTINETTI P., et al., Detailed design optimization of the MITICA negative ion accelerator in view of the ITER NBI, Nucl. Fusion 56 (2016) 016015.

[19] ASUNTA O., et al., Computer Physics Communications 188 (2015) 3346 